\title{
On the ampleness of the normal bundle of line congruences
}

\author{
Enrique Arrondo, Marina Bertolini and Cristina Turrini
}

Communicated by Karl Strambach

\begin{abstract}
In this paper we study the normal bundle of the embedding of subvarieties of dimension $n-1$ in the Grassmann variety of lines in $\mathbb{P}^{n}$. Making use of some results on the geometry of the focal loci of congruences ([4] and [5]), we give some criteria to decide whether the normal bundle of a congruence is ample or not. Finally we apply these criteria to the line congruences of small degree in $\mathbb{P}^{3}$.
\end{abstract}

Keywords. Normal bundle, ampleness, line congruence, Grassmannian.

2000 Mathematics Subject Classification. 14J60.

\section{Introduction}

One of the most powerful tools to understand the geometry of the embedding of a subvariety inside a bigger ambient space is to study the normal bundle of the embedding. For example, deformations of the embedding correspond to sections of the normal bundle. The case in which the ambient variety is a projective space has been studied thoroughly in the literature. As a sample of the interest of the normal bundle of projective varieties, the Harsthorne conjecture is related to the splitting of the normal bundle, and the ampleness of the normal bundle provides a simple proof of Barth-Larsen theorem by using Le Potier vanishing theorem for ample vector bundles ([15]).

However, when the ambient variety is not a projective space, few things are known. For example, Ballico ([9]) has studied the ampleness of the normal bundle of curves in quadrics, while Papantonopoulou ([14]) and Goldstein ([10], [11]) restricting their attention to the four-dimensional quadric identified with the Grassmann variety of lines in $\mathbb{P}^{3}$, have studied not only curves, but also surfaces (i.e. congruences of lines). Recently, new results in the flavor of Barth-Larsen's have been proved for subvarieties in other ambient spaces, like Grassmannians of lines (see [6]).

Also, some analogue to Hartshorne's conjecture in codimension two has been stated for these Grassmannians (see [7]). In order to approach these new prob- 
lems, it becomes crucial to have a good understanding of the normal bundle of subvarieties of Grassmannians of lines.

The goal of this paper is to reinterpret and extend Goldstein and Papantonopoulou's results to arbitrary congruences of lines, i.e. to subvarieties of dimension $n-1$ in the Grassmann variety of lines in $\mathbb{P}^{n}$. The main point is that, in the same way as the projectivization of the normal bundle of projective subvarieties is the conormal variety, the projectivization of the normal bundle of congruences is closely related to their focal loci. Since we have already studied the geometry of the focal loci of congruences ([4] in case $n=3$ and [5] in general), we can give criteria to decide whether the normal bundle of a congruence is ample or not.

We start in Section 2 by recalling several well-known facts about Grassmannians of lines, which however are not easy to find in the literature, and that we will use throughout the paper. In particular, we give a geometric description of the tangent bundle of a Grassmannian, its projectivization and the map induced by the tautological line bundle of this projectivization.

In Section 3, we give the geometrical interpretation of the projectivization of the normal bundle of some subvarieties of Grassmannians of lines. In the concrete case of line congruences, we recall from [5] the main results about focal loci of line congruences, and relate them with the projectivization of the normal bundle (see Theorem 3.7). As in [5], most of the results are obtained by means of local computations.

In Section 4, using the description given in the previous section, we study the ampleness of the normal bundle of congruences. More precisely, we prove (see Proposition 4.1) that the normal bundle is not ample if and only if there is a onedimensional family of lines of the congruence all of them sharing the same focal pair of point and hyperplane (we call this family a non-ampleness curve). In the particular case of congruences of lines in $\mathbb{P}^{3}$, we recover the result of Goldstein and Papantonopoulou stating that such a non-ampleness curve must be a pencil of lines. However, we show that, when $n>3$, we could have infinitely many non-ampleness curves, none of them pencils (see Example 4.5), and also higher dimensional non-ampleness loci (see Proposition 4.6). We end this section with Theorem 4.8, in which we give a more practical criterion, in terms of local coordinates, to decide whether a particular curve is a non-ampleness curve.

Finally, in Section 5, we go through the list of line congruences of degree at most six in $\mathbb{P}^{3}$ and apply the previous results to decide in which cases the normal bundle is ample, at least for the general congruence. The main idea is that the only possible non-ampleness curves are necessarily pencils of lines, so that for each congruence it is enough to apply Theorem 4.8 to each pencil of the congruence (when there is no other easier way of studying the ampleness). We acknow- 
ledge the fruitful use in this section of the computer algebra system Macaulay by D. Bayer and M. Stillman.

\section{Generalities on Grassmannians}

Let $k$ be an algebraically closed field of characteristic zero. If $V$ is a vector space over $k$, we will denote by $\mathbb{P}(V)$ the set of rank-one quotients of $V$. More generally, we will denote by $G(m, n)$ the Grassmann variety of $m$-dimensional linear subspaces of $\mathbb{P}(V)$ over $k$, i.e. the set of rank- $(m+1)$ quotients of $V$. We will deal mainly with the case $m=1$, and we will usually just write $G$ instead of $G(1, n)$. We will fix (dual) bases for $V$ and $V^{*}$ and denote by $\left\{p_{i j}\right\}_{0 \leq i<j \leq n}$ the usual Plücker coordinates in $G$, so that we will regard $G$ as a subvariety of $\mathbb{P}\left(\bigwedge^{2} V\right)$. Although we will always assume $i<j$, it will be useful sometimes to write some $p_{i j}$ with $j \leq i$, which should be interpreted as $-p_{j i}$ (or zero if $i=j$ ).

Convention. In order to distinguish whether we refer to a subspace as a subset of $\mathbb{P}^{n}$ or as an element of the Grassmannian, we will use the following convention: small letters $l$ will represent points of the Grassmannian $G(m, n)$, while the corresponding capital letters $L$ will represent the $k$-dimensional linear subspace $L \subset \mathbb{P}^{n}$ represented by $l$ and $\vec{l} \subset V^{*}$ will stand for the $(m+1)$-dimensional linear subspace defining $L$.

Regarded as a vector bundle (we will interchange freely the notions of locally free sheaf and vector bundle), the universal quotient bundle can be interpreted as the dual of the subbundle $\breve{Q}$ of the trivial vector bundle $G \times V^{*}$ consisting of the pairs $(l, v)$ such that $v$ is a vector of the linear space $\vec{l}$ defining $L$. We will also consider the universal vector bundle $S:=G \times V^{*} / \breve{Q}$. It can be also regarded as the dual of the subbundle of $G \times V^{*}$ consisting of the pairs $(l, h)$ such that $H$ vanishes on $L$.

For each line $L$ with Plücker coordinates $\bar{a}:=\left(a_{i j}\right)$ we define the vectors

$$
w_{i}=\left(a_{i 0}, \ldots, a_{i n}\right)
$$

(for $i=0, \ldots, n$ ) and the linear forms

$$
H_{i j k}=a_{j k} x_{i}-a_{i k} x_{j}+a_{i j} x_{k}
$$

(for $i, j, k=0, \ldots, n$ ). Observe that, with this notation, the vectors $w_{i}$ span the linear space of $\vec{l} \subset V^{*}$, while the linear forms $H_{i j k}$ span the linear subspace $H^{0}\left(\mathbb{P}^{n}, d_{L, \mathbb{P}^{n}}(1)\right) \subset V$ (in fact, the equations of $G$ in its Plücker ambient space are precisely $H_{i j k}\left(w_{l}\right)=0$ for $\left.i, j, k, l=0, \ldots, n\right)$. 
We include now the following well-known facts about $G$, of which we will prove in detail the last part, because it is not easy to find in the literature and because we will need the precise details appearing in the proof.

Lemma 2.1. Let $U_{i j}$ be the subset of $G$ consisting of those lines for which the Plïcker coordinate $p_{i j}$ is not zero. Then:

(i) $U_{i j}$ is an affine open set of $G$ with affine coordinates $\frac{p_{i k}}{p_{i j}}, \frac{p_{j k}}{p_{i j}}$ with $k=$ $0, \ldots, \hat{i}, \ldots, \hat{j}, \ldots, n$.

(ii) On each $U_{i j}$, there is a trivialization of $\check{\mathscr{Q}}$ given by $w_{i}, w_{j}$ in the sense that there is an isomorphism $U_{i j} \times k^{2} \cong \check{\mathscr{Q}}_{\mid U_{i j}}$, where a pair $(\bar{a},(\lambda, \mu))$ is identified with the element $\left(\bar{a}, \lambda w_{i}+\mu w_{j}\right) \in \check{Q}$. Similarly, there is a trivialization of $\breve{s}$ given by the $H_{i j k}$, with $k=0, \ldots, \hat{i}, \ldots, \hat{j}, \ldots, n$.

(iii) There is an isomorphism $\check{\mathcal{Q}} \otimes \check{S} \cong \Omega_{G}$ determined on each open subset $U_{i j}$ by $\left(\bar{a}, w_{i} \otimes H_{i j k}\right) \mapsto a_{i j}^{2} d\left(\frac{p_{i k}}{p_{i j}}\right)$ and $\left(\bar{a}, w_{j} \otimes H_{i j k}\right) \mapsto a_{i j}^{2} d\left(\frac{p_{j k}}{p_{i j}}\right)$.

(iv) The tautological $\mathcal{O}(1)$ over the projective bundle $\mathbb{P}\left(T_{G}\right)$ defines a morphism

$$
\varphi: \mathbb{P}\left(T_{G}\right) \rightarrow \mathbb{P}\left(V \otimes V^{*}\right)
$$

such that the fiber at each $l \in G$ maps isomorphically onto the linear span, after the Segre embedding, of $L \times \mathbb{P}\left(H^{0}\left(\mathbb{P}^{n}, d_{L, \mathbb{P}^{n}}(1)\right)^{*}\right) \subset \mathbb{P}^{n} \times \mathbb{P}^{n *}$.

Proof. Part (i) is standard, while part (ii) follows easily from the fact that any line in $U_{i j}$ is determined by just $w_{i}, w_{j}$, and dually by just the $H_{i j k}$ with $k \neq i, j$. Part (iii) follows from a tedious but straightforward computation showing that the morphisms we defined at each $U_{i j}$ glue together.

For part (iv), we first recall that the map $G \times V \otimes V^{*} \rightarrow T_{G}=\mathcal{Q} \otimes S$ induces an epimorphism from $V \otimes V^{*}$ onto the space of global sections of $T_{G}$ (its kernel being the set of homoteties of $\left.V^{*}\right)$. Hence $\mathbb{P}\left(T_{G}\right)$ can be regarded as a projective subbundle of $G \times \mathbb{P}\left(V \times V^{*}\right)$ with the same $\mathcal{O}(1)$, which on $G \times \mathbb{P}\left(V \times V^{*}\right)$ defines the projection onto the second factor. The proof concludes by observing that (iii) is saying that the fiber of $\mathbb{P}\left(T_{G}\right)$ at a point of coordinates $\bar{a}$ becomes, inside $\mathbb{P}\left(V \times V^{*}\right)$, the subspace generated by the products $w_{i} \otimes H_{i j k}, w_{j} \otimes H_{i j k}$, with $k=0, \ldots, \hat{i}, \ldots, \hat{j}, \ldots, n$, which is (after projectivizing) the Segre ambient space of the product of the space generated by $w_{i}, w_{j}$ (which yields $L$ ) and the space generated by the $H_{i j k}$ (which yields $\left.\mathbb{P}\left(H^{0}\left(\mathbb{P}^{n}, \mathcal{l}_{L, \mathbb{P}^{n}}(1)\right)^{*}\right)\right)$.

Remark 2.2. Since $V \otimes V^{*}$ is isomorphic to $\operatorname{End}(V)$, we can think of the elements of $\mathbb{P}\left(V \times V^{*}\right)$ as endomorphisms of $V^{*}$ (up to multiplication by a constant). For 
instance, following Lemma 2.1, in the open set $U_{01}$, if $L$ is the line spanned by the two points $\left(0: a_{01}: \cdots: a_{0 n}\right)$ and $\left(-a_{01}: 0: a_{12}: \cdots: a_{1 n}\right)$, the image via $\varphi$ of the element $\left(l, d\left(\frac{p_{0 i}}{p_{01}}\right)\right)$ is the endomorphism given by the matrix

$$
\left(\begin{array}{ccccccc}
0 & 0 & 0 & \ldots & 0 & \ldots & 0 \\
\frac{a_{1 i}}{a_{01}} & -\frac{a_{0 i}}{a_{01}} & 0 & \ldots & 1 & \ldots & 0 \\
\vdots & \vdots & \vdots & & \vdots & & \vdots \\
\frac{a_{0 n} a_{1 i}}{a_{01}{ }^{2}} & -\frac{a_{0 n} a_{0 i}}{a_{01}{ }^{2}} & 0 & \ldots & \frac{a_{0 n}}{a_{01}} & \ldots & 0
\end{array}\right)
$$

and the image of $\left(l, d\left(\frac{p_{1 i}}{p_{01}}\right)\right)$ is given by the matrix

$$
\left(\begin{array}{ccccccc}
\frac{a_{1 i}}{a_{01}} & -\frac{a_{0 i}}{a_{01}} & 0 & \ldots & 1 & \ldots & 0 \\
0 & 0 & 0 & \ldots & 0 & \ldots & 0 \\
\vdots & \vdots & \vdots & & \vdots & & \vdots \\
\frac{a_{1 n} a_{1 i}}{a_{01}{ }^{2}} & -\frac{a_{1 n} a_{0 i}}{a_{01}{ }^{2}} & 0 & \ldots & \frac{a_{1 n}}{a_{01}} & \ldots & 0
\end{array}\right)
$$

With this identification, we have the following result (which was indicated to us by Jorge Caravantes):

Corollary 2.3. Identify an element $A \in \mathbb{P}\left(V \otimes V^{*}\right)$ with an endomorphism of $V^{*}$. Then:

(i) If $A=\varphi(\zeta)$, with $\zeta \in \mathbb{P}\left(T_{G}\right)$ in the fiber of a line $l \in G$, then the twodimensional linear subspace $\vec{l} \subset V^{*}$ contains the image of the endomorphism $A$ and is contained in its kernel; in particular $\operatorname{rk}(A) \leq 2$.

(ii) If $A$ is the image by $\varphi$ of infinitely many elements of $\mathbb{P}\left(T_{G}\right)$, then $\operatorname{rk}(A)=1$; i.e. $A=(p, h) \in \mathbb{P}(V) \times \mathbb{P}\left(V^{*}\right)$.

Proof. A pair $(p, h) \in \mathbb{P}(V) \times \mathbb{P}\left(V^{*}\right)$ is identified with an endomorphism of $V^{*}$ vanishing on $\vec{H}$ and having as image the one-dimensional linear subspace of $\vec{p} \subset V^{*}$. By Lemma 2.1, the element $A$ is a linear combination of endomorphisms with image in $\vec{l}$ and vanishing on $\vec{l}$, and hence (i) follows right away.

Suppose now $A$ has rank two, and let us show that it cannot be the image by $\varphi$ of infinitely many elements of $\mathbb{P}\left(T_{G}\right)$, which will prove (ii). If $A=\varphi(\zeta)$, with $\zeta \in \mathbb{P}\left(T_{G}\right)$, by (i) $\zeta$ is necessarily in the fiber of the line $L$ defined by the image of $A$ (which by assumption has dimension two). Finally, Lemma 2.1 implies that the fiber of the line $l$ maps injectively into $\mathbb{P}\left(V \otimes V^{*}\right)$, so the result follows. 


\section{The projective normal bundle and the focal loci of line congruences}

In this section we use the results obtained in the previous one to give a geometric description of the projectivization of the normal bundle of subvarieties of $G(1, n)$. We will concentrate on the case of line congruences (see definition below) and on the relation of this theory with the theory of focal loci.

We start with very particular subvarieties of $G(1, n)$ that will be very useful in the next section.

Proposition 3.1. Let $X \subset \mathbb{P}^{n}$ be an $r$-dimensional variety and let $L \subset \mathbb{P}^{n}$ be a line meeting $X$ transversally at a smooth point $p \in X$. Let also $\Sigma \in G(1, n)$ be the set of lines of $\mathbb{P}^{n}$ meeting $X$ and write also $N$ for the normal sheaf of $\Sigma$ in $G(1, n)$. Then, with the notation of Lemma 2.1, if $l$ is a smooth point of $\Sigma$, the

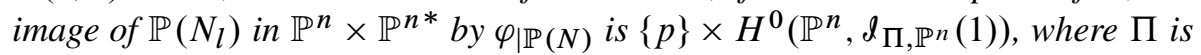
the linear span $\Pi$ of $L$ and the tangent space to $X$ at $p$.

Proof. Choose coordinates in $\mathbb{P}^{n}$ such that $p=(1: 0: \cdots: 0), L: x_{2}=$ $\cdots=x_{n}=0$, and the tangent space to $X$ at $p$ is $T_{p} X: x_{1}=\cdots=x_{n-r}=0$ (hence $\Pi: x_{2}=\cdots=x_{n-r}=0$ ), and take the corresponding Plücker coordinates. In this way, the point $p$ is the origin in the affine coordinates $x_{1}, \ldots, x_{n}$ of $\left\{x_{0}=1\right\}$ and the line $L$ becomes the origin when we take the affine coordinates $p_{02}, \ldots, p_{0 n}, p_{12}, \ldots, p_{1 n}$ of $U_{01}=\left\{p_{01}=1\right\}$. Choose now a local parametrization of $X$ at the origin

$$
\left\{\begin{aligned}
x_{1} & =f_{1}\left(u_{1}, \ldots, u_{r}\right) \\
& \vdots \\
x_{n-r} & =f_{n-r}\left(u_{1}, \ldots, u_{r}\right) \\
x_{n-r+1} & =u_{1} \\
\vdots & \\
x_{n} & =u_{r}
\end{aligned}\right.
$$

where $u_{1}, \ldots, u_{r}$ is a system of local parameters of $X$ at $p$, and $f_{1}, \ldots, f_{n-r}$ have order at least two. In this way, a local parametrization of $\Sigma$ around $l$ is given by assigning to the parameters $u_{1}, \ldots, u_{r}, v_{1}, \ldots, v_{n-1}$ the line spanned by the rows of the matrix

$$
\left(\begin{array}{cccccccc}
1 & f_{1} & f_{2} & \ldots & f_{n-r} & u_{1} & \ldots & u_{r} \\
0 & 1 & v_{1} & \ldots & v_{n-r-1} & v_{n-r} & \ldots & v_{n-1}
\end{array}\right)
$$


or, in the above affine Plücker coordinates,

$$
\begin{array}{ll}
p_{0 i}=v_{i-1} & \text { for } i=2, \ldots, n, \\
p_{1 i}=f_{1} u_{i-1}-f_{i} & \text { for } i=2, \ldots, n-r, \\
p_{1 i}=f_{1} u_{i-1}-u_{i-n+r} & \text { for } i=n-r+1, \ldots, n .
\end{array}
$$

This proves that the tangent space to $\Sigma$ at $l$ has equations $p_{12}=\cdots=p_{1, n-r}=$ 0 . By Lemma 2.1, $d p_{1 i}$ is identified, at the origin, with the tensor $w_{1} \otimes H_{01 i}$, i.e. the pair $\left(p, H_{i}\right) \in \mathbb{P}^{n} \times \mathbb{P}^{n *}$, where $H_{i}: x_{i}=0$. Since $H_{2}, \ldots, H_{n-r}$ generate $H^{0}\left(\mathbb{P}^{n}, d_{\Pi, \mathbb{P}^{n}}(1)\right)$, the result follows.

Remark 3.2. In the statement of Proposition 3.1, the hypothesis that $l$ is a smooth point of $\Sigma$ is not strictly necessary. One could have that $L$ meets $X$ in two different points, which would provide two different branches of $\Sigma$, and our statement remains true for the normal sheaf of any of the branches. Observe also that, as our local computations implicitly showed, the smoothness of $\Sigma$ at $l$ already implies that $L$ and $X$ meet transversally. A similar observation can be made about the smoothness of $X$ at $p$, in which we could only impose to have smooth branches.

Definition 3.3. By a line congruence we shall mean an $(n-1)$-dimensional subvariety (which we will always assume to be smooth and irreducible) of $G=G(1, n)$.

For a given a line congruence $Y$, we recall the following definitions and results from [5]:

(i) Let $I_{Y}^{0} \subset Y \times \mathbb{P}^{n}$ be the incidence variety consisting of the pairs $(p, l)$ for which $l$ is an element of $Y$ and $p$ is a point of the line $L$ and let $q_{0}: I_{Y}^{0} \rightarrow \mathbb{P}^{n}$ be the second projection. The focal locus of $Y$ will be the branch locus $F$ of $q_{0}$, and the elements of $F$ will be called focal points. If $(p, l)$ is in the ramification locus of $q_{0}$ we will say that $p$ is a focal point for the line L. Obviously a fundamental point, i.e. a point through which there pass infinitely many lines of the congruence, is a focal point.

(ii) Let $I_{Y}^{2} \subset Y \times G(2, n)$ be the incidence variety consisting of the pairs $(l, \pi)$ for which $l$ is a line of $Y$ and $\Pi$ is a plane containing the line $L$ and let $q_{2}: I_{X}^{2} \rightarrow G(2, n)$ be the second projection (which is not surjective if $n \geq 4$ ). The locus of focal planes of $Y$ will be the branch locus $F_{2}$ of $q_{2}$, and if $(l, \pi)$ is in the ramification locus of $q_{2}$ we will say that $\Pi$ is a focal plane for the line $L$. As before, a fundamental plane, i.e. a plane containing infinitely many lines of the congruence, is a focal plane.

(iii) Let $I_{Y}^{n-1} \subset Y \times \check{\mathbb{P}}^{n}$ be the incidence variety consisting of pairs $(l, h)$ such that the hyperplane $H$ contains the line $L$. Let $q_{n-1}: I_{Y}^{n-1} \rightarrow \check{\mathbb{P}}^{n}$ be the second 
projection (which has no finite fibers if $n \geq 4$ ). The locus of focal hyperplanes $F_{n-1}$ is the branch locus of $q_{n-1}$. If $(l, h)$ is in the ramification locus of $q_{n-1}$ then we will say that $H$ is a focal hyperplane for the line $L$.

We summarize here the main results from [5] that we will need:

Lemma 3.4. Either all the points of a line $L$ are focal (in which case we will say that $L$ is a focal line) or $L$ contains $n-1$ focal points (counted with multiplicity). Similarly, when the number of focal planes (resp. hyperplanes) of $L$ is finite, this number is $n-1$, and in this case they are in general position (in the sense that the $n-1$ focal planes span $\mathbb{P}^{n}$ and the intersection of the $n-1$ focal hyperplanes is $L$ ).

Lemma 3.5. The different focal loci are related as follows:

(i) Let $I_{Y}^{0,2} \subset Y \times \mathbb{P}^{n} \times G(2, n)$ be the incidence variety consisting of the triples $(p, l, \pi)$ for which $l$ is an element of $Y, p$ is a point of $L$ and $\Pi$ is a plane containing the line $L$. Then the branch locus of the projection $q_{0,2}: I_{Y}^{0,2} \rightarrow \mathbb{P}^{n} \times G(2, n)$ maps surjectively under the two projections to the focal locus and the locus of focal planes of $Y$. Moreover, if for a line $L$ there is only a finite number of pairs $(p, \pi)$ (called focal pairs for $L$ ) such that $(p, l, \pi)$ is a ramification point of $q_{0,2}$, then this number of pairs is $n-1$.

(ii) If a line $l$ has exactly $n-1$ focal pairs $\left(p_{1}, \pi_{1}\right), \ldots,\left(p_{n-1}, \pi_{n-1}\right)$, then $l$ has exactly $n-1$ focal hyperplanes, which are $H_{1}, \ldots, H_{n-1}$, where $H_{i}$ is the span of all the focal planes except $\pi_{i}$ (we will say that the pairs $\left(p_{i}, h_{i}\right)$ are focal dual pairs). Moreover, $H_{i}$ is the tangent hyperplane to the focal locus at the point $p_{i}$.

We also recall from [5] the way of computing the focal loci of a congruence $Y$ in local coordinates. We will fix a line $L$ of the congruence and a point $p \in L$, a plane $\Pi \supset L$ and a hyperplane $H \supset L$ and we want to characterize when they are focal for $L$.

We fix coordinates in $\mathbb{P}^{n}$ such that the line $L$ has equations $x_{2}=\cdots=$ $x_{n}=0$. Hence the open set $U_{01}$ of $G$ is the affine set in which the coordinates $p_{02}, \ldots, p_{0 n}, p_{12}, \ldots, p_{1 n}$ represent the line spanned by the rows of the matrix

$$
\left(\begin{array}{ccccc}
0 & 1 & p_{02} & \ldots & p_{0 n} \\
-1 & 0 & p_{12} & \ldots & p_{1 n}
\end{array}\right)
$$

and the origin in these coordinates is precisely the line $L$. Thus, a local parametrization of $Y$ around $l$ is given by sending the set of local parameters $u_{1}, \ldots, u_{n-1}$ 
to the line spanned by the rows of the matrix

$$
\left(\begin{array}{ccccc}
0 & 1 & h_{2} & \ldots & h_{n} \\
-1 & 0 & k_{2} & \ldots & k_{n}
\end{array}\right)
$$

with $h_{i}=h_{i}\left(u_{1}, \ldots, u_{n-1}\right), k_{i}=k_{i}\left(u_{1}, \ldots, u_{n-1}\right)$ regular functions vanishing at the origin.

Lemma 3.6. With the above choice of coordinates:

(i) The point $p$ is focal for $L$ if and only if the matrix

$$
\left(\begin{array}{ccc}
\frac{\partial k_{2}}{\partial u_{1}}(0) & \cdots & \frac{\partial k_{n}}{\partial u_{1}}(0) \\
\vdots & \ddots & \vdots \\
\frac{\partial k_{2}}{\partial u_{n-1}}(0) & \cdots & \frac{\partial k_{n}}{\partial u_{n-1}}(0)
\end{array}\right)
$$

has rank at most $n-2$ (i.e. its determinant is zero).

(ii) The plane $\Pi$ is focal for $L$ if and only if the matrix

$$
\left(\begin{array}{cccccc}
\frac{\partial k_{3}}{\partial u_{1}}(0) & \ldots & \frac{\partial k_{n}}{\partial u_{1}}(0) & \frac{\partial h_{3}}{\partial u_{1}}(0) & \ldots & \frac{\partial h_{n}}{\partial u_{1}}(0) \\
\vdots & & \vdots & \vdots & & \vdots \\
\frac{\partial k_{3}}{\partial u_{n-1}}(0) & \ldots & \frac{\partial k_{n}}{\partial u_{n-1}}(0) & \frac{\partial h_{3}}{\partial u_{n-1}}(0) & \ldots & \frac{\partial h_{n}}{\partial u_{n-1}}(0)
\end{array}\right)
$$

has rank at most $n-2$.

(iii) The pair $(p, \pi)$ is a focal pair for $L$ if and only if the matrix

$$
\left(\begin{array}{cccccc}
\frac{\partial k_{2}}{\partial u_{1}}(0) & \ldots & \frac{\partial k_{n}}{\partial u_{1}}(0) & \frac{\partial h_{3}}{\partial u_{1}}(0) & \ldots & \frac{\partial h_{n}}{\partial u_{1}}(0) \\
\vdots & & \vdots & \vdots & & \vdots \\
\frac{\partial k_{2}}{\partial u_{n-1}}(0) & \ldots & \frac{\partial k_{n}}{\partial u_{n-1}}(0) & \frac{\partial h_{3}}{\partial u_{n-1}}(0) & \ldots & \frac{\partial h_{n}}{\partial u_{n-1}}(0)
\end{array}\right)
$$

has rank at most $n-2$.

(iv) The hyperplane $H$ is focal for $L$ if and only if the matrix

$$
\left(\begin{array}{cc}
\frac{\partial k_{n}}{\partial u_{1}}(0) & \frac{\partial h_{n}}{\partial u_{1}}(0) \\
\vdots & \vdots \\
\frac{\partial k_{n}}{\partial u_{n-1}}(0) & \frac{\partial h_{n}}{\partial u_{n-1}}(0)
\end{array}\right)
$$

has rank at most one. 
(v) If $L$ has a finite number of focal pairs, the pair $(p, h)$ is a focal dual pair for $L$ if and only if $\frac{\partial k_{n}}{\partial u_{1}}(0)=\cdots=\frac{\partial k_{n}}{\partial u_{n-1}}(0)=0$.

Proof. It follows from Section 4 in [5], observing that now we are writing $h_{i}$ instead of $g_{i}$ and $-k_{i}$ instead of $f_{i}$, and also we need only to evaluate at the origin so the expressions in [5] simplify. In this way, matrices of (i), (ii) and (iii) are respectively matrices $B, C$ and $B C$ (after removing repetitions) of Section 4.1, and the matrix of (iv) is matrix $B^{\prime}$ of Section 4.2. For (v), see the end of the proof of Theorem 4.1 in [5].

We can finally relate the projective normal bundle with the focal loci.

Notation. From now on, when no confusion can arise, for a line congruence $Y$ we will denote by $N$ the normal bundle of $Y$ in $G=G(1, n)$. Regarding $\mathbb{P}(N)$ as a subset of $\mathbb{P}\left(T_{G}\right)$ we will also denote by $\varphi_{Y}$ the restriction to $\mathbb{P}(N)$ of the map $\varphi: \mathbb{P}\left(T_{G}\right) \rightarrow \mathbb{P}\left(V \otimes V^{*}\right)$ defined in Lemma 2.1 (which is thus the map associated to $\left.\mathcal{O}_{\mathbb{P}(N)}(1)\right)$.

Theorem 3.7. Let $Y \subseteq G(1, n)$ be a line congruence with normal bundle $N$ and let $\eta \in \mathbb{P}(N)$ be an element in the fiber $\mathbb{P}\left(N_{l}\right)$ of $l \in Y$. Then:

(i) If $\varphi_{Y}(\eta)$ is a pair $(p, h) \in \mathbb{P}(V) \times \mathbb{P}\left(V^{*}\right)$, then $p$ is a focal point for $l$ and $H$ is a focal hyperplane for $L$.

(ii) If $L$ is a line with exactly $n-1$ focal pairs, then $\varphi_{Y}(\eta)$ is a pair $(p, h)$ if and only $(p, h)$ is a focal dual pair of $L$. In particular, in this case the image by $\varphi_{Y}$ of the fiber of $\mathbb{P}(N)$ over l is the span of the $n-1$ focal dual pairs of $L$.

Proof. We choose coordinates and notation as for Lemma 3.6. An element of $\mathbb{P}(N) \subset \mathbb{P}\left(T_{G}\right)$ can be regarded as the class (up to multiplication by a constant) of the restriction to $Y$ of a non-zero form

$$
\omega=\alpha_{2} d\left(\frac{p_{02}}{p_{01}}\right)+\cdots+\alpha_{n} d\left(\frac{p_{0 n}}{p_{01}}\right)+\beta_{2} d\left(\frac{p_{12}}{p_{01}}\right)+\cdots+\beta_{n} d\left(\frac{p_{1 n}}{p_{01}}\right)
$$

with $\alpha_{2}, \ldots, \alpha_{n}, \beta_{2} \ldots, \beta_{n} \in k$. The form $\omega$ is in the conormal bundle $N^{*}$ if and only if it is an element of the kernel of the natural morphism $\pi: \Omega_{G \mid Y} \rightarrow \Omega_{Y}$ that maps $d\left(\frac{p_{0 i}}{p_{01}}\right)$ to $d h_{i}$ and $d\left(\frac{p_{1 i}}{p_{01}}\right)$ to $d k_{i}$. Hence $\omega$ is in the kernel of $\pi$ if and only if for $i=1, \ldots, n-1$

$$
\alpha_{2} \frac{\partial h_{2}}{\partial u_{i}}(0)+\cdots+\alpha_{n} \frac{\partial h_{n}}{\partial u_{i}}(0)+\beta_{2} \frac{\partial k_{2}}{\partial u_{i}}(0)+\cdots+\beta_{n} \frac{\partial k_{n}}{\partial u_{i}}(0)=0 .
$$


By Remark 2.2, and having in mind that the affine Plücker coordinates of $l$ are all zero, the endomorphism given by $\varphi(\omega)$ is represented by the matrix

$$
\left(\begin{array}{ccccc}
0 & 0 & \beta_{2} & \ldots & \beta_{n} \\
0 & 0 & \alpha_{2} & \ldots & \alpha_{n} \\
0 & 0 & 0 & \ldots & 0 \\
\vdots & \vdots & \vdots & & \vdots \\
0 & 0 & 0 & \ldots & 0
\end{array}\right)
$$

which corresponds to $(p, H)$ if and only if $\alpha_{2}=\cdots=\alpha_{n}=\beta_{2}=\cdots=\beta_{n-1}=0$. This is equivalent, by (3.4), to $\frac{\partial k_{n}}{\partial u_{1}}(0)=\cdots=\frac{\partial k_{n}}{\partial u_{n-1}}(0)=0$, which together with Lemma 3.6 proves the theorem.

\section{General results on the ampleness of the normal bundle of congruences}

From what we have seen so far we can readily prove the following result:

Proposition 4.1. Let $Y \subseteq G(1, n)$ be a line congruence with normal bundle $N$. Then $N$ is not ample if and only if there exists a curve $C \subset Y$, a point $p \in \mathbb{P}^{n}$ and a hyperplane $H \ni p$ such that for any $l \in C$, the pair is focal dual for $L$. In particular, $C$ is contained in the Schubert variety $\Omega(p, H)$ of lines passing through a point $p$ and contained in $H$.

Proof. We need to characterize when the map $\varphi_{Y}$ contracts some curve $C$ to a point of $\mathbb{P}\left(V \otimes V^{*}\right)$. By Corollary 2.3, such a $C$ can only be contracted to a point of the form $(p, H)$. The result follows now from Theorem 3.7.

Definition 4.2. We will call a curve as in Proposition 4.1 a non-ampleness curve.

In the particular case $n=3$, since $\Omega(p, H)$ is a line inside $G(1,3)$, the only non-ampleness curves are necessarily lines (Goldstein in [10] called them ampleness lines, but we found this name misleading, so we did not take it). In this case, we recover from Proposition 4.1 the following result of Papantonopoulou (see [14] Proposition 2.4) and Goldstein (see [10] Proposition 4.4):

Corollary 4.3. Let $Y$ be a surface in $G(1,3)$. Then the normal bundle of $Y$ is not ample if and only if there exists a pencil of lines $\Omega(p, \Pi) \subset Y$ such that for any $l \in \Omega(p, \Pi)$ the pair $(p, \pi)$ is focal dual for $Y$. 
Remark 4.4. It is important to notice that, if $n=3$, the notions of focal planes and focal hyperplanes coincide, but the notions of focal pair and focal dual pair are completely different. In fact, according to Lemma 3.5 , if $\left(x_{1}, \pi_{1}\right),\left(x_{2}, \pi_{2}\right)$ are the focal pairs of a line, then the focal dual pairs of the line are $\left(x_{1}, \pi_{2}\right),\left(x_{2}, \pi_{1}\right)$.

When $n$ is arbitrary, the criterion of Proposition 4.1 is not easy to check because of two reasons. First, there are too many curves to look at as possible candidates for non-ampleness curves (although the philosophy should be that if the congruence is "sufficiently general" it should not contain curves contained in any Schubert variety $\Omega(p, H)$ ). On the other hand, once a candidate is found, it is not easy a priory to check whether it satisfies the condition of Proposition 4.1. To solve this last problem, we will give at the end this section a characterization in terms of local equations (see Theorem 4.8).

The first difference with the case $n=3$ is that the non-ampleness curves do not need to be pencils of lines. We give next an example showing that in fact there could be infinitely many non-ampleness curves, none of them a pencil of lines.

Example 4.5. Let $Q \subset \mathbb{P}^{4}$ be a smooth quadric hypersurface and let $Y \subset G(1,4)$ be the congruence of lines contained in $Q$. It is well know (see for instance [3]) that $Y$ is, after the Plücker embedding, the double Veronese embedding of $\mathbb{P}^{3}$. In fact, $Y$ is the zero locus of the section of $S^{2} Q$ corresponding to the choice of the quadric $Q$. In particular its normal bundle is $N=S^{2} Q_{\mid Y}$. By Proposition 4.1, any possible non-ampleness curve for $Y$ should be a curve in $Y$ such that all of its lines pass through a common point $p$. Clearly, $p$ should be a point of $Q$, and the only possibility is that such non-ampleness curve is the set $C$ of all the lines contained in $Q$ and passing through $p$, i.e. the lines in the quadric cone obtained by intersection $Q$ with its tangent hyperplane at $p$. This means that $C$ is isomorphic to $\mathbb{P}^{1}$ and $\mathcal{Q}_{\mid C}=\mathcal{O}_{\mathbb{P}^{1}} \oplus \mathcal{O}_{\mathbb{P}^{1}}$ (2). This implies that $N_{\mid C}=\mathcal{O}_{\mathbb{P}^{1}} \oplus \mathcal{O}_{\mathbb{P}^{1}}(2) \oplus \mathcal{O}_{\mathbb{P}^{1}}$ (4), and hence such $C$ is always a non-ampleness curve.

Another phenomenon when $n>3$ is that the non-ampleness locus could have dimension bigger than one. An example of this is provided by the following result, which provides a non-ampleness criterion for certain congruences.

Proposition 4.6. Let $Y \subset G(1, n)$ be a line congruence such that there exists $X \subset \mathbb{P}^{n}$ satisfying that through the general point of $X$ there passes an $s$-dimensional family of lines of $Y$. Then, for a general point $p \in X$ and a general hyperplane $H \subset \mathbb{P}^{n}$ containing the tangent space to $X$ at $p$, the fiber of the pair $(p, h)$ under $\varphi_{Y}$ has dimension at least $s-1$. In particular, if $s>1$ then the normal bundle of $Y$ is not ample. 
Proof. By assumption, the set of lines of $Y$ passing through $p$ has dimension $s$. Hence those of them contained also in $H$ form a family of dimension $s-1$. By Proposition 3.1, for a general $L$ in this family, the pair $(p, h)$ is in the image of $\varphi_{Y}$.

Remark 4.7. The hypothesis of Proposition 4.6 can be satisfied even in the most special case, i.e. when $X$ is a curve and $s=n-1$. In this case, there is a complete classification of these congruences (see [2]). More precisely, for any $n$, there are infinitely many cases in which $X$ is a line and a finite number of cases in which $X$ is another curve.

We end this section with the promised characterization of when a given curve is a non-ampleness curve in terms of a local parametrization of the congruence, which we will use in the next section.

Theorem 4.8. Let $Y \subseteq G(1, n)$ be a line congruence and let $C$ be a curve in $Y$ contained in a Schubert variety $\Omega(p, H)$ and let $l$ be a smooth point of $C$. Choose coordinates in $\mathbb{P}^{n}$ such that $p=(1: 0: \ldots: 0), l$ is the line of equations $x_{2}=x_{3}=\cdots=x_{n}=0$ and $H$ has equation $x_{n}=0$. Take local coordinates $u_{1}, u_{2}, \ldots, u_{n-1}$ for $Y$ near $l$ such that the local ideal of $C$ in $Y$ is $\left(u_{1}, u_{2}, \ldots, u_{n-2}\right)$. Then, $C$ is a non-ampleness curve if and only if $p_{1 n}\left(u_{1}, u_{2}, \ldots, u_{n-1}\right) \in\left(u_{1}, u_{2}, \ldots, u_{n-2}\right)^{2}$.

Proof. We just repeat the computations of the proof of Theorem 3.7, but moving $l$ along the curve $C$. We thus fix the same system of coordinates and parametrizations. Since $C$ is contained in $\Omega(p, H)$, we have now that $h_{n}, k_{2}, k_{3}, \ldots, k_{n} \in$ $\left(u_{1}, u_{2}, \ldots, u_{n-2}\right)$.

The fact that $C$ is a non-ampleness curve is equivalent to the fact that, for any element of $C,(p, h)$ is a focal dual pair for it. We thus fix $\bar{u}=\left(0, \ldots, 0, \bar{u}_{n-1}\right)$, which defines the line of $L(\bar{u}) \subset \mathbb{P}^{n}$ spanned by the rows of

$$
\left(\begin{array}{cccccc}
0 & 1 & \bar{h}_{2} & \ldots & \bar{h}_{n-1} & 0 \\
-1 & 0 & 0 & \ldots & 0 & 0
\end{array}\right),
$$

where $\bar{h}_{i}=h_{i}(\bar{u})$. Performing the change of coordinates in $\mathbb{P}^{n}$

$$
\begin{gathered}
x_{0}^{\prime}=x_{0}, \quad x_{1}^{\prime}=x_{1}, \quad x_{2}^{\prime}=-\bar{h}_{2} x_{1}+x_{2}, \quad \ldots, \\
x_{n-1}^{\prime}=-\bar{h}_{n-1} x_{1}+x_{n-1}, \quad x_{n}^{\prime}=x_{n},
\end{gathered}
$$

we have that $u_{1}, \ldots, u_{n-1}$ represent the line spanned by the points whose coordi- 
nates are the rows of

$$
\left(\begin{array}{cccccc}
0 & 1 & h_{2}-\bar{h}_{2} & \ldots & h_{n-1}-\bar{h}_{n-1} & h_{n} \\
-1 & k_{2} & 0 & \ldots & k_{n-1} & k_{n}
\end{array}\right) .
$$

Now $p, L(\bar{u}), H$ are in the conditions of Theorem 3.7 (up to a translation of $u_{n-1}$ by $\left.\bar{u}_{n-1}\right)$. Hence $(p, h)$ is a focal dual pair for $L(\bar{u})$ if and only if $\frac{\partial k_{n}}{\partial u_{1}}(\bar{u})=$ $\cdots=\frac{\partial k_{n}}{\partial u_{n-1}}(\bar{u})=0$. Hence $C$ is a non-ampleness curve if and only if we have $\frac{\partial k_{n}}{\partial u_{1}}, \ldots, \frac{\partial k_{n}}{\partial u_{n-1}} \in\left(u_{1}, \ldots, u_{n-2}\right)$, which is equivalent to $k_{n} \in\left(u_{1}, \ldots, u_{n-2}\right)^{2}$, which proves the theorem.

Remark 4.9. We will use Theorem 4.8 mainly for congruences in $G(1,3)$ with a finite number of pencils of lines. In this case, it will be enough in general to use the theorem for just one pencil of the congruence instead of all of them. Indeed, assume we have an irreducible family $\mathscr{H}$ of congruences such that the general one contains a finite number of pencils of lines. Let $\mathbb{F}_{0,2}$ denote the flag variety parametrizing the pencils of lines in $\mathbb{P}^{3}$. We consider the incidence variety $I \subset \mathbb{F}_{0,2} \times \mathscr{H}$ consisting of the pairs $(\Omega, Y)$ such that the congruence $Y$ contains the pencil $\Omega$. We write $p_{1}, p_{2}$ for the projections from $I$ to $\mathbb{F}_{0,2}$ and $\mathscr{H}$ respectively. If $\mathscr{H}$ has the property that the set of congruences of $\mathscr{H}$ containing a given $\Omega$ is irreducible, this implies that $I$ is also irreducible (because all the fibers of $p_{1}$ are isomorphic to each other). The fact that the general congruence of $\mathscr{H}$ contains a finite number of pencils of lines is equivalent to say that $p_{2}$ is generically finite. Suppose that we have found a congruence $Y \in \mathscr{H}$ with a pencil $\Omega$ which is not a non-ampleness line. This means that the dimension of the closed set $B \subset I$ of pairs $(\Omega, Y)$ such that $\Omega$ is a non-ampleness line for $Y$ is strictly smaller that the dimension of $I$. Since $p_{2}$ is generically finite, this implies that the image of $B$ is not the whole $\mathscr{H}$. Therefore, the general congruence of $\mathscr{H}$ does not contain non-ampleness lines, and hence its normal bundle is ample.

\section{Congruences of lines in $\mathbb{P}^{3}$ of small degree}

We collect first in the following table the description of the congruences of degree $d \leq 6$ (see for instance [8]). Afterwards we analyze case by case these congruences in order to determine whether the normal bundle of these congruences is ample or not. In the last column of the table we give the answer for the generic element of the corresponding Hilbert scheme.

As it is clear, by duality (a congruence of lines in $\mathbb{P}^{3}$ yields a congruence of lines in $\check{\mathbb{P}}^{3 *}$ ), we can restrict our analysis to the case $a \leq b$. 


\begin{tabular}{|c|c|c|c|c|c|}
\hline & $(a, b)$ & $\pi$ & Description of $Y$ & Embedding & $\begin{array}{c}N_{Y / G(1,3) \text { am- }} \\
\text { ple for generic } \\
\text { element? }\end{array}$ \\
\hline \hline 1 & $(0,1)$ & 0 & $\mathbb{P}^{2}$ & $|L|$ & No \\
\hline 2 & $(1,1)$ & 0 & $\mathbb{P}^{1} \times \mathbb{P}^{1}$ & $\left|C_{0}+F\right|$ & Yes \\
\hline 3 & $(1,2)$ & 0 & blow up of $\mathbb{P}^{2}$ in one point & $|2 L-E|$ & No \\
\hline 4 & $(1,3)$ & 0 & Veronese embedding of $\mathbb{P}^{2}$ & $|2 L|$ & Yes \\
\hline 5 & $(2,2)$ & 0 & Rational normal scroll $\mathbb{F}_{0}$ & $\left|C_{0}+2 F\right|$ & No \\
\hline 6 & $(2,2)$ & 0 & Rational normal scroll $\mathbb{F}_{2}$ & $\left|C_{0}+3 F\right|$ & Yes \\
\hline 7 & $(2,2)$ & 1 & blow up of $\mathbb{P}^{2}$ in five points & $\left|3 L-E_{1}-\cdots-E_{5}\right|$ & Yes \\
\hline 8 & $(2,3)$ & 1 & blow up of $\mathbb{P}^{2}$ in four points & $\left|3 L-E_{1}-\cdots-E_{4}\right|$ & Yes \\
\hline 9 & $(2,3)$ & 2 & blow up of $\mathbb{P}^{2}$ in eight points & $\left|4 L-2 E_{1}-E_{2}-\cdots-E_{8}\right|$ & No \\
\hline 10 & $(3,3)$ & 1 & elliptic scroll $\mathbb{X}_{0}$ & $\left|C_{0}+\mathscr{L} F\right|$, deg $\mathscr{L}=3$ & Yes \\
\hline 11 & $(3,3)$ & 1 & blow up of $\mathbb{P}^{2}$ in three points & $\left|3 L-E_{1}-\cdots-E_{3}\right|$ & Yes \\
\hline 12 & $(3,3)$ & 2 & blow up of $\mathbb{P}^{2}$ in seven points & $\left|4 L-2 E_{1}-E_{2}-\cdots-E_{7}\right|$ & Yes \\
\hline 13 & $(3,3)$ & 4 & $\mathrm{~K} 3$ surface & & \\
\hline
\end{tabular}

Table 1. Congruences of degree $d \leq 6$.

First we recall some standard facts:

Remark 5.1. When $Y$ is a surface in $G(1,3)$, it is easy to show the ampleness of the normal bundle $N=N_{Y / G(1,3)}$ in the following cases:

(i) $Y$ is the complete intersection of a linear complex of degrees $d_{1}$ (i.e. the intersection in $\mathbb{P}^{5}$ of the Grassmannian $G(1,3)$ with a hypersurface of degree $d_{1}$ ) and a linear complex of degree $d_{2}$, because in this case we have $N=\mathcal{O}_{Y}\left(d_{1}\right) \oplus \mathcal{O}_{Y}\left(d_{2}\right)$.

(ii) more generally $Y$ is the complete intersection of the Grassmannian with a smooth codimension two subvariety $Z \subset \mathbb{P}^{5}$ because in this case $N=$ $N_{Z, \mathbb{P}^{5} \mid Y}$ and $N_{Z, \mathbb{P}^{5}}$ is ample.

(iii) $Y$ is the zero locus of a section of $Q\left(d_{1}\right)$ or $S\left(d_{2}\right)$ with $d_{1}, d_{2}>0$.

We are now ready for our case-by-case analysis:

Cases 2, 7, 13. The normal bundle $N_{Y / G(1,3)}$ of congruences 2, 7, 13 listed above are ample by Remark 5.1 (i), since these congruences are complete intersections.

Case 4. In this case, the normal bundle is ample, since the Veronese surface has no lines. 
Cases 8, 12. In case 8, the normal bundle is also ample by Remark 5.1 (iii) since the congruence is the zero locus of a section of the ample bundle $Q(1)$. In case 12 , the ampleness of the normal bundle follows from Remark 5.1 (ii) and the description given in [8] of this congruence as complete intersection of the Grassmannian with a Segre variety.

Case 1. In this case, we apply Proposition 4.6 to the dual congruence, which consists of the set of lines passing through one point.

Case 3. Following [8] the congruence $Y$ is obtained as follows: given a conic $\Gamma$ in a plane $\Lambda$ and a line $L_{0}$ not contained in $\Lambda$ intersecting $\Gamma$ in a point $p_{0}, Y$ is the closure of the set of lines meeting $\Gamma$ and $L$ in different points. The scroll structure is given by the pencils of lines $\Omega(p, \Pi)$, with $p \in \Gamma$ and $\Pi=\left\langle L_{0}, p\right\rangle$. Observe that when $p=p_{0}$ the plane $\Pi$ is the plane $\Pi_{0}$ spanned by $L$ and the tangent line at $p_{0}$ to $\Gamma$. Since $a=1$, the focal locus of the congruence is the fundamental locus, i.e. $\Gamma \cup L_{0}$. Notice also that the planes $\Pi$ are fundamental, hence focal, although there are also other focal planes. Hence for any $l \in \Omega\left(p_{0}, \Pi_{0}\right) \backslash\left\{l_{0}\right\}$, the point $p_{0}$ is its only focal point, while $\Pi_{0}$ is a focal plane. Therefore the pair $\left(p_{0}, \pi_{0}\right)$ is focal dual for $Y$. This, by Corollary 4.3 , implies that $\left(p_{0}, \pi_{0}\right)$ is a non-ampleness line. A similar reasoning allows to reprove Goldstein's statement (see [11]) that this is the only non-ampleness line on $Y$.

Case 10. We recall the description of this elliptic scroll as a congruence, following [12]. Let us consider in $\mathbb{P}^{3}$ an elliptic cubic curve $D$ contained in a plane $\Lambda$ and endowed with the standard group law, i.e. the sum of three points is zero if and only if they are collinear. Fix a point $\sigma \in D$, another point $R \in \Lambda \backslash D$ and a point $\kappa \in \mathbb{P}^{3} \backslash \Lambda$. To any point $u \in D$ one associates a line $L(u)$ as follows: first one considers the point $-u+2 \sigma \in D$ and the line $r(u)$ joining it with $R$. Denoting by $A$ and $B$ the two other points of intersection of $r(u)$ and $D$, it follows that

$$
(-u+2 \sigma)+A+B=0,
$$

so that the three points $u,-A-\sigma,-B-\sigma$ lie on a same line, which we call $L(u)$. The congruence is thus given by the pencils of lines through $u$ in the planes $\Pi(u)$ spanned by $\kappa$ and $L(u)$. From this construction, it is immediate to see that all points of $D$ are fundamental and hence focal for the congruence and similarly the planes $\Pi(u)$ are fundamental and focal. Moreover the planes $\Pi(u)$ are tangent to the focal surface $F$ of the congruence (which is a cone with vertex $\kappa$, as it can be seen by duality). For a general element $l \in \Omega(u, \Pi(u))$ of the congruence, the focal points for $L$ are $u$ and a point $p \in F$ such that the tangent plane to $F$ at $p$ is $\Omega(u, \Pi(u))$. The corresponding focal planes are $\Pi(u)$ and (see Lemma 2.2 and Lemma 4.1 of [4]) the plane spanned by $L$ and the tangent line to $D$ at $u$. 
Notice that, if the line $L(u)$ is the tangent line to $D$ at $u$, these two focal planes coincide, and hence $\Omega(u, \Pi(u))$ is a non-ampleness line. The existence of a nonampleness line is therefore equivalent to the existence of a point $u \in D$ such that either $-A-\sigma=u$ or $-B-\sigma=u$. Now we will prove that such situation happens for six points on $D$, in other words that there are six non-ampleness lines in the congruence. The point $u$ is univocally determined from equation (5.1) by giving $A, B \in D$ such that the line they span passes through $R$. Hence we are looking for pairs $A, B$ that are collinear with $R$ and satisfy $2 A+B=-3 \sigma$ (or symmetrically $A+2 B=-3 \sigma)$. To find these pairs of points we consider the intersection in the product $C \times C$ of the divisor $X=\{(A, B) \mid A, B$ and $R$ are collinear $\}$ with the divisor $Y=\{(A, B) \mid 2 A+B=-3 \sigma\}$. The number $n_{0}$ of the intersection points does not change if we substitute $Y$ with $Y^{\prime}=\{(A, B) \mid 2 A+B=0\}$ since $Y$ and $Y^{\prime}$ are two different fibers of the map from $C \times C \rightarrow C$ defined by $(A, B) \mapsto 2 A+B$. Hence $n_{0}$ is the number of tangent lines to $C$ through $R$, which is 6 .

In the rest of the cases we will use the local criterion given by Theorem 4.8.

Cases 5, 6. We first recall their geometric descriptions from [12]. Case 5 is obtained by giving an automorphism $\psi$ of a plane conic $\Gamma$ and a point $p$ outside the plane spanned by $\Gamma$. The congruence consists thus of the union of the pencils $\Omega\left(p_{t}, \Pi_{t}\right)$ determined by a point $q_{t}$ of $\Gamma$ and the plane $\Pi_{t}$ spanned by $p, q_{t}$ and $\psi\left(q_{t}\right)$. Case 6 (which is omitted in [8]) is a degeneration of case 5 in which $p$ belongs to $\Gamma$. Hence, it will be enough to prove the non-ampleness for case 5. More precisely, we will prove that, for any fixed point $q_{0}$ of $\psi$ (which always exists) the pencil $\Omega\left(q_{0}, \Pi_{0}\right)$ is a non-ampleness line. Observe that we could take $\psi$ to be the identity, in which case we obtain infinitely many non-ampleness pencils. This is precisely the particular example given in Section $\S 3$ of [11], which is proved in [10] to be the only one, together with case 1 , with infinitely many non-ampleness curves.

Now, in order to apply Theorem 4.8 to prove the non-ampleness in case 5, we take a fixed point $q_{0}$ of $\psi$ and take coordinates such that $q_{0}=(1: 0: 0: 0)$, $p=(0: 0: 1: 0)$ and $\Gamma$ is the conic parametrized by $\left(1: t: 0: t^{2}\right)$ on the plane $\Pi_{0}: x_{2}=0$. With these equations, $\psi$ is given by $t^{\prime}=a t /(c t+d)$, with $a d \neq 0$, and for $q_{t}=\left(1: t: 0: t^{2}\right) \in \Gamma$, the plane $\Pi_{t}$ has equation

$$
x_{0} t^{2}-x_{1}(1+c t+d) t+x_{3}(c t+d)=0 \text {. }
$$

Introducing an affine parameter $u$ for the pencils $\Omega\left(q_{t}, \Pi_{t}\right)$, we get a local parametrization of the congruence such that for any value of the parameters $t, u$, its 
corresponding line is the one generated by the rows of the matrix

$$
\left(\begin{array}{cccc}
1 & 0 & -t u & \frac{t^{2}}{c t+d} \\
0 & 1 & u & \frac{t(1+c t+d)}{c t+d}
\end{array}\right)
$$

with $d \neq 0$. In particular, in this parametrization $p_{13}=-\frac{t^{2}}{c t+d} \in\left(t^{2}\right)$, which proves by Theorem 4.8 that $\Omega\left(q_{0}, \Pi_{0}\right)$ is a non-ampleness line.

In the last cases we need the extra the aid of Maple, in order to apply Theorem 4.8. Moreover, we will prove the ampleness of the normal bundle for a particular congruence. Since the Hilbert scheme of these congruences is irreducible (see [8]), this will prove the ampleness of the normal bundle for the general congruence. However, we will need to check that our particular choice of the congruence is smooth, and we do so with the aid of the computer algebra system Macaulay.

Case 9. In this case, the congruence is a Castelnuovo surface given by the blow up of $\mathbb{P}^{2}$ in eight points, embedded by the linear system $\left|4 L-2 E_{1}-E_{2}-\cdots-E_{8}\right|$ of the quartics with a double point $P_{1}$ and seven simple points $P_{2}, \ldots, P_{8}$. This surface contains only a finite numbers of lines, which are the seven exceptional divisors $E_{2}, \ldots, E_{8}$ and the seven curves in the linear systems $\left|L-E_{1}-E_{2}\right|, \ldots$, $\left|L-E_{1}-E_{8}\right|$. We will prove that $N_{Y / G(1,3)}$ is ample by checking that none of these lines of the surface is a non-ampleness line. We will do so only for the line $E_{2}$, since we can apply the argument of Remark 4.9.

To see that we are in the conditions of Remark 4.9, we recall from [8] the construction of the congruences of this family $\mathscr{H}$. We fix a line $L \subset \mathbb{P}^{3}$, a point $p \in L$ and a cubic complex $\Sigma$ containing the $\alpha$-plane $\alpha(p)$ of lines passing through $p$. Then the congruence is the residual congruence of $\alpha(p)$ in the complete intersection of $\Sigma$ and the linear complex of all the lines of $\mathbb{P}^{3}$ meeting $L$. We have thus that all the lines of the congruence meet $L$ (i.e. $L$ is a fundamental line). More precisely, for each point of $L$, the lines of the congruence passing through it form a cubic cone, while for each plane containing $L$ the set of lines of the congruence contained in it forms a curve of degree two (we have to remove the pencil of lines through $p$ ). The latter endows the congruence with a conic fibration structure over $L$. In terms of the Castelnuovo surface, the linear complex of lines meeting $L$ is given by $\left|3 L-E_{1}-E_{2}-\cdots-E_{8}\right|+\left|L-E_{1}\right|$, the fundamental line $L$ corresponds to the base point of $\left|3 L-E_{1}-E_{2}-\cdots-E_{8}\right|$, and the conic fibration is given by $\left|L-E_{1}\right|$. The seven singular conics of the fibration are thus the seven pairs of lines $\left(E_{i}, L-E_{1}-E_{i}\right)$. In particular, all the lines contained in the congruence correspond to pencils of lines $\Omega(q, \Pi)$ such that the plane $\Pi$ contains the line $L$. Hence, the set of congruences of $\mathscr{H}$ containing a fixed $\Omega(q, \Pi)$ 
is parametrized by the choice of a line $L$ contained in $\Pi$, a point $p \in L \backslash\{q\}$ and a cubic complex $\Sigma$ containing $\alpha(p)$ and $\Omega(q, \Pi)$. Since this is an irreducible parametrization, it follows from Remark 4.9 that it is enough to check that $E_{2}$ is not a non-ampleness line.

In order to apply Theorem 4.8, we choose coordinates $u, x, y$ in $\mathbb{P}^{2}$ and take $P_{1}=(1: 0: 0), P_{2}=(0: 0: 1), P_{3}=(0: 1: 0), P_{4}=(1: 1: 1), P_{5}=$ $(1: 2:-1), P_{6}=(1:-1: 2), P_{7}=(1:-2: 3), P_{8}=(1:-3:-2)$. Choosing five suitable quartics generating the linear system $\left|4 L-2 E_{1}-E_{2}-\cdots-E_{8}\right|$ we get a parametrization

$$
\begin{aligned}
p_{01} & =276 u y^{3}-138 u^{2} x y-276 u x^{2} y-207 x^{2} y^{2}+207 x y^{3}+138 u^{2} y^{2}, \\
p_{02} & =276 u x y^{2}-876 u^{2} x y+1056 u x^{2} y+870 x^{2} y^{2}+78 x y^{3}-1404 u^{2} y^{2}, \\
p_{03}= & 0, \\
p_{12}= & 276 x^{3} y+920 u^{2} x y-2760 u x^{2} y-2300 x^{2} y^{2}+4416 u^{2} y^{2}-552 u^{2} x^{2}, \\
p_{13}= & -19044 x^{3} y+12696 u^{2} x y+19044 x^{2} y^{2}-12696 u^{2} x^{2} \\
& \quad-25392 u x^{3}+25392 u x y^{2}, \\
p_{23}= & 80040 x^{3} y-129168 u^{2} x y+25392 u x^{2} y+7176 x^{2} y^{2} \\
& \quad-80592 u^{2} x^{2}+97152 u x^{3},
\end{aligned}
$$

yielding an embedding of the Castelnuovo surface in $G(1,3)$, which we checked to be smooth. In the affine set $y=1$ with coordinates $u, x$, the origin is the point $P_{2}$, and the blow-up at it is defined locally by affine coordinates $u, \lambda$, where $x=\lambda u$, and $u=0$ is the equation of the exceptional divisor $E_{2}$. Hence the congruence is locally parametrized by the expressions $p_{i j}(u, \lambda u, 1) / u$. Thus one sees that $E_{2}$ is the line $p_{03}=p_{12}=p_{13}=p_{23}=0$, which corresponds to the pencil of lines in the plane $x_{3}=0$ with center in the point $(1: 0: 0: 0)$ in $\mathbb{P}^{3}$. Since also $p_{13}(u, \lambda u, 1) / u \notin(u)^{2}$, it follows from Theorem 4.8 that $E_{2}$ is not a non-ampleness line.

Case 11. This case can be dealt with in the same way as above. This time the congruence is a Del Pezzo surface given by the blow up of $\mathbb{P}^{2}$ in three points, embedded in $\mathbb{P}^{5}$ by a five-dimensional linear subsystem of $\left|3 L-E_{1}-E_{2}-E_{3}\right|$ of the cubics through three points $P_{1}, P_{2}, P_{3}$. This surface contains only a finite numbers of lines which are the three exceptional divisors $E_{1}, E_{2}, E_{3}$ and the three curves in the linear systems $\left|L-E_{1}-E_{2}\right|,\left|L-E_{1}-E_{3}\right|,\left|L-E_{2}-E_{3}\right|$. As in case 9 , to prove that $N_{Y / G(1,3)}$ is ample, it is enough to check that $E_{2}$ is not a non-ampleness line. Indeed the congruences of this family $\mathscr{H}$ are given (see [8]) as zero loci of general sections of a vector bundle $\dot{E}(2)$, where the bundle $\dot{E}$ varies 
in an irreducible moduli space. Hence, for any pencil of lines $\Omega \subset G(1,3)$, the family of congruences of $\mathscr{H}$ containing $\Omega$ is irreducible, since it is parametrized by the choice of a vector bundle $\dot{E}$ and a general section of $\dot{E} \otimes I_{\Omega}(2)$. Therefore Remark 4.9 allows us to study only the line $E_{2}$.

We can assume, after choosing coordinates $u, x, y$ in $\mathbb{P}^{2}$, that $P_{1}=(1,0,0)$, $P_{2}=(0,0,1), P_{3}=(0,1,0)$. We take, as a five-dimensional subsystem of $\left|3 L-E_{1}-E_{2}-E_{3}\right|$, the one generated by

$$
\begin{aligned}
& p_{01}=\frac{44}{14579}\left(403235800 x y u+139030672 \sqrt{5} x y u+1603263760 x u^{2}\right. \\
& -16765850 x y^{2}-816239885 y u^{2}-1992930575 u x^{2} \\
& +399435100 y x^{2}+16765850 u y^{2}+5073492 \sqrt{5} u y^{2} \\
& +138073820 \sqrt{5} x^{2} y+553174278 \sqrt{5} x u^{2}-5073492 \sqrt{5} x y^{2} \\
& \left.-281287894 \sqrt{5} y u^{2}-688021548 \sqrt{5} u x^{2}\right) \text {, } \\
& p_{02}=-\frac{44}{14579}\left(403235800 x y u-1232651700 u x^{2}+139030672 \sqrt{5} x y u\right. \\
& +207883345 x u^{2}-8382925 x y^{2}+12269905 y u^{2} \\
& +214409775 y x^{2}+72089470 \sqrt{5} x^{2} y+71540994 \sqrt{5} x u^{2} \\
& \left.-2536746 \sqrt{5} x y^{2}+5528498 \sqrt{5} y u^{2}-424683560 \sqrt{5} u x^{2}\right), \\
& p_{03}=-\frac{44}{61}\left(10198 \sqrt{5} x u^{2}+31640 x u^{2}+26018 \sqrt{5} y u^{2}+57135 y u^{2}\right. \\
& \left.-40376 \sqrt{5} u x^{2}-123125 u x^{2}+4160 \sqrt{5} x^{2} y+34350 y x^{2}\right), \\
& p_{12}=-\frac{1936}{3484381}\left(2190303550 x y u-13714794150 u x^{2}+755189332 \sqrt{5} x y u\right. \\
& +8656778415 x u^{2}-3652404540 y u^{2}+4329813175 y x^{2} \\
& +1641552458 \sqrt{5} x^{2} y+2969880482 \sqrt{5} x u^{2}-1467465176 \sqrt{5} y u^{2} \\
& \left.-4654346428 \sqrt{5} u x^{2}\right) \text {, } \\
& p_{13}=-\frac{3872}{14579}\left(4582225 x y u-22911125 u x^{2}+1579894 \sqrt{5} x y u\right. \\
& +18328900 x u^{2}-9164450 y u^{2}+4582225 y x^{2}+1579894 \sqrt{5} x^{2} y \\
& \left.+6319576 \sqrt{5} x u^{2}-3159788 \sqrt{5} y u^{2}-7899470 \sqrt{5} u x^{2}\right), \\
& p_{23}=\frac{1936}{14579}\left(u^{2}-6 u x+2 y u+x y\right) x(620 \sqrt{5}+7031)(174 \sqrt{5}+575) \text {. }
\end{aligned}
$$


One checks that this yields a smooth surface in $G(1,3)$. Also, as in case 9, the substitutions $p_{i j}(u, \lambda u, 1)$ give a parametrization of the congruence with parameters $u, \lambda$ such that $u=0$, which is the local equation of $E_{2}$, corresponds to the pencil of lines in the plane $x_{3}=0$ with center in the point $(1: 0: 0: 0)$. Since $p_{13}(u, \lambda u, 1) / u \notin(u)^{2}$, it follows from Theorem 4.8 that $E_{2}$ is not a nonampleness line.

\section{Bibliography}

[1] E. Arrondo, Line congruences of low order, Milan Journal of Mathematics (formerly Rendiconti del Seminario Matematico e Fisico di Milano) 70 (2002), 223-243.

[2] E. Arrondo, M. Bertolini and C. Turrini, Classification of smooth congruences with a fundamental curve, in: Projective Geometry with Applications, pp. 43-56, Lecture Notes in Pure and Applied Mathematic 166, Marcel Dekker, 1994.

[3] E. Arrondo, M. Bertolini and C. Turrini, Congruences of small degree in $G(1,4)$, Comm. Algebra 26 (1998), 3249-3266.

[4] E. Arrondo, M. Bertolini and C. Turrini, A focus on focal surface, Asian J. of Math. 5 (2001), 535-560.

[5] E. Arrondo, M. Bertolini and C. Turrini, Focal loci in $G(1, N)$, Asian J. of Math. 9 (2005), 449-472.

[6] E. Arrondo and J. Caravantes, On the Picard group of low-codimension subvarieties, Indiana U. Math. J. 58 (2009), 1023-1050.

[7] E. Arrondo and M. L. Fania, Evidence to subcanonicity of codimension two submanifolds of G(1, 4), Internat. J. Math. 17 (2006), 157-168.

[8] E. Arrondo and I. Sols, On congruences of lines in the projective space, Mém. Soc. Math. France, 50, Société Mathématique de France, 1992, Supplément au Bulletin de la Société Mathématique de France, tome 120, fascicule 3.

[9] E. Ballico, Normal bundle to curves in quadrics, Bull. Soc. Math. France 109 (1981), $227-235$.

[10] N. Goldstein, A special surface in the 4-quadric, Duke Math. J. 50 (1983), 745-761.

[11] N. Goldstein, Examples of non-ample normal bundles, Comp. Math 51 (1984), 189-192.

[12] N. Goldstein, Scroll surfaces in Gr (1,3), Rend. Sem. Mat. Univers. Politecn. Torino, Special issue (1987), 69-75.

[13] R. Hartshorne, Algebraic Geometry, Graduate Texts in Mathematics 52, SpringerVerlag, New York, Heidelberg, Berlin, 1977. 
[14] A. Papantonopoulou, Surfaces in the Grassmann variety $G(1,3)$, Proc. Amer. Math. Soc. 77 (1979), 15-18.

[15] M. Schneider and J. Zintl, The theorem of Barth-Lefschetz as a consequence of Le Potier's vanishing theorem, Manuscripta Math. 80 (1993), 259-263.

Received June 16, 2008; revised March 13, 2009.

\section{Author information}

Enrique Arrondo, Departamento de Álgebra, Facultad de Matemáticas, Universidad Complutense de Madrid, 28040 Madrid, Spain.

E-mail: enrique_arrondo@mat.ucm.es

Marina Bertolini, Dipartimento di Matematica "F. Enriques", Università degli Studi di Milano, via C. Saldini 50, 20133 Milan, Italy.

E-mail: marina.bertolini@unimi.it

Cristina Turrini, Dipartimento di Matematica “F. Enriques”, Università degli Studi di Milano, via C. Saldini 50, 20133 Milan, Italy.

E-mail: cristina.turrini@unimi.it 
Copyright of Forum Mathematicum is the property of De Gruyter and its content may not be copied or emailed to multiple sites or posted to a listserv without the copyright holder's express written permission. However, users may print, download, or email articles for individual use. 